\title{
Anomalous scalings of cuprate strange metals from nonlinear electrodynamics
}

\author{
Sera Cremonini, Anthony Hoover, Li Li, and Steven Waskie \\ Department of Physics, Lehigh University, Bethlehem, Pennsylvania, 18018 USA
}

(Received 28 December 2018; published 29 March 2019)

\begin{abstract}
We examine transport in a holographic model which describes, through a nonlinear gauge field sector, generic nonlinear interactions between the charge carriers. Scaling exponents are introduced by using geometries which are nonrelativistic and hyperscaling-violating in the infrared. In the dilute charge limit in which the gauge field sector does not backreact on the geometry, a particularly simple nonlinear theory reproduces the anomalous temperature dependence of the resistivity and Hall angle of the cuprate strange metals, $R \sim T$ and $\cot \Theta_{H} \sim T^{2}$ while also allowing for a linear entropy $S \sim T$, and predicts that the magnetoresistance for small values of the magnetic field $h$ should scale as $\sim h^{2} T^{-4}$. Our study lends evidence to the idea that the strange metal behavior of the cuprates relies crucially on the linear temperature dependence of the entropy.
\end{abstract}

DOI: 10.1103/PhysRevD.99.061901

\section{INTRODUCTION}

The anomalous metallic state in the high-temperature superconducting cuprates is one of the most remarkable puzzles in condensed matter physics [1]. The transport properties of the strange metal phase are characterized by universal temperature scalings which are robust across widely different systems, and are believed to be controlled by an underlying strongly interacting quantum critical sector. Magnetotransport measurements have identified anomalous power law behavior $\sim T^{n}$ for the in-plane resistivity, the Hall angle and the weak-field magnetoresistance (corresponding to $n=1,2$ and -4 , respectively) [2-4]. The universality of these scaling laws, seen in a variety of materials, strongly motivates the search for robust physical mechanisms that can reproduce such observations.

The holographic gauge/gravity duality has shown to be a useful theoretical laboratory to probe the physics of strongly interacting quantum critical states, by mapping them onto a dual gravitational problem $[5,6]$. Using the techniques of holography, in this paper we will study a model that describes a sector of probe charge carriers interacting amongst themselves and with a larger set of neutral quantum critical degrees of freedom (d.o.f.). Thus far standard EinsteinMaxwell-dilaton (EMD) theories have been unable to reproduce the anomalous scalings of the strange metal phase in the presence of a magnetic field (see e.g., Refs. [7-10]). Indeed, there is evidence [11-13] that to reliably capture

Published by the American Physical Society under the terms of the Creative Commons Attribution 4.0 International license. Further distribution of this work must maintain attribution to the author(s) and the published article's title, journal citation, and DOI. Funded by SCOAP . transport in these phases it may be crucial to take into account the nontrivial dynamics between the charge d.o.f. This is reasonable given that we are dealing with strongly correlated electron matter, and was already suggested in Ref. [14].

With this in mind, we examine a holographic theory which includes a generic nonlinear gauge field sector and compute the associated direct current (DC) conductivities. We consider a particularly simple nonlinear model whose structure is natural from the point of view of the DiracBorn-Infeld (DBI) action. Remarkably, this simple, solvable model provides the first holographic realization of the temperature scalings of the entropy $\sim T$, resistivity $\sim T$, Hall angle $\sim T^{2}$ and weak-field magnetoresistance $\sim T^{-4}$ observed in the cuprates-with a minimal set of assumptions. The underlying mechanism relies on having a quantum critical IR fixed point and on the nonlinear structure of the interactions between the charges. Our results also suggest that the strange metal behavior is intimately tied to the linear temperature dependence of the entropy.

\section{HOLOGRAPHIC SETUP AND CONDUCTIVITIES}

We are interested in describing a strongly coupled quantum theory containing a sector of dilute charge carriers that interact amongst themselves as well as with a quantum critical bath. The charge d.o.f. should be thought of as a probe when compared to the larger set of neutral quantum critical d.o.f. What we have in mind are gravitational theories of the type

$$
S=\int d^{4} x \sqrt{-g}\left[\mathcal{L}_{\mathrm{Bath}}+\mathcal{L}_{\mathrm{U}(1)}\right]
$$


with a bath sector $\mathcal{L}_{\text {Bath }}$ supported, for example, by a neutral scalar $\phi$ and axionic scalars, and a charge sector $\mathcal{L}_{\mathrm{U}(1)}$ describing the dynamics of a $U(1)$ gauge field $A_{\mu}$. In particular, since we are interested in capturing generic nonlinear electrodynamics effects, the latter will be encoded in the Lagrangian term $\mathcal{L}_{\mathrm{U}(1)}=\mathcal{L}(s, p, \phi)$, which is a generic function of the two combinations

$$
s=-\frac{1}{4} F_{\mu \nu} F^{\mu \nu}, \quad p=-\frac{1}{8} \epsilon_{\mu \nu \rho \sigma} F^{\mu \nu} F^{\rho \sigma},
$$

with $F_{\mu \nu}$ the field strength for $A_{\mu}$ and $\epsilon_{\mu \nu \rho \sigma}$ the covariant Levi-Civita tensor, and allows for couplings between the gauge field and the neutral scalar $\phi$. Such theories include as a special case the DBI model studied in Refs. $[13,15]$ and extend those studied in Ref. [16] by adding a scalar sector.

Assuming a background which is homogeneous and isotropic, the quantum critical bath can be described holographically using a black brane geometry of the form

$$
d s^{2}=-U(r) d t^{2}+\frac{d r^{2}}{U(r)}+C(r)\left(d x^{2}+d y^{2}\right),
$$

with a nontrivial scalar $\phi=\phi(r)$ depending on the holographic radial coordinate $r$. The holographic DC conductivities associated with the conserved current $J^{\mu}$ dual to $A_{\mu}$ can be obtained following the prescription developed by Ref. [17] (see also Ref. [16]). In the probe limit the DC conductivity matrix $\sigma_{i j}$ for the broad class of theories (1) is only sensitive to the structure of $\mathcal{L}(s, p, \phi)$ and in particular is given by

$$
\sigma_{x x}=\mathcal{L}^{(1,0,0)}, \quad \sigma_{x y}=-\mathcal{L}^{(0,1,0)},
$$

where we have defined for convenience

$$
\mathcal{L}^{(1,0,0)} \equiv \frac{\partial \mathcal{L}(s, p, \phi)}{\partial s}, \quad \mathcal{L}^{(0,1,0)} \equiv \frac{\partial \mathcal{L}(s, p, \phi)}{\partial p} .
$$

The corresponding resistivity and Hall angle are then

$$
\begin{aligned}
R_{x x} & =\frac{\sigma_{x x}}{\sigma_{x x}^{2}+\sigma_{x y}^{2}}=\frac{\mathcal{L}^{(1,0,0)}}{\left(\mathcal{L}^{(1,0,0)}\right)^{2}+\left(\mathcal{L}^{(0,1,0)}\right)^{2}}, \\
\cot \Theta_{H} & =\frac{\sigma_{x x}}{\sigma_{x y}}=-\frac{\mathcal{L}^{(1,0,0)}}{\mathcal{L}^{(0,1,0)}},
\end{aligned}
$$

where it should be understood that all functions are evaluated at the horizon $r=r_{h}$ of the black brane (3) whose temperature is $T=U^{\prime}\left(r_{h}\right) / 4 \pi$.

Note that when $\mathcal{L}^{(0,1,0)}=0, \quad \sigma_{x y}=0$ and hence $\tan \Theta_{H}=0$. Thus, the presence of $p \sim F \wedge F$ in the theory leads to a distinctively different behavior for the conductivities. As an example, in the standard linear EMD theory $\mathcal{L}(s, p, \phi)=Z(\phi) s$ thus far it has been difficult to realize the scaling behavior of the cuprates. In the probe limit this situation is not ameliorated, because although the associated resistivity $R_{x x}=1 / Z$ can in principle be engineered to be linear, the Hall conductivity is trivial. This compels us to study nonlinear electrodynamics effects.

Finally, we stress that the result (6) is quite general, as it relies only on a minimal set of assumptions-a homogeneous and isotropic metric (3) modeling the quantum critical bath, and the presence of a dilute set of charge carriers. The analysis of the DC conductivities away from the probe limit is included in the Supplemental Material [18], where it can be seen that $\sigma_{i j}$ in the backreacted case is sensitive not only to the gauge field sector, but also to the geometry and the structure of the model supporting the quantum critical bath. Interestingly, we find that the probe limit results can be obtained from the fully backreacted case when the scale of momentum dissipation dominates over the other physical scales in the system.

\section{QUANTUM CRITICAL BATH GEOMETRY}

Motivated by condensed matter studies of quantum criticality in strange metals [21-23], we will be specifically interested in solutions that are nonrelativistic and violate hyperscaling in the IR of the geometry-thus, the dual system will be quantum critical in a generalized sense. To work with the standard holographic dictionary we consider geometries which asymptote to anti-de Sitter (AdS) at the boundary. The IR scaling behavior of such geometries will lead naturally to clean scaling regimes in the holographic transport coefficients and in particular in the DC conductivities, which are determined by horizon data.

One advantage of working in the probe limit is that we have a clean separation between the background geometry and the gauge field sector. In particular, a simple holographic model which supports analytical scaling geometries contains a dilatonic scalar $\phi$ and two axionic scalars $\psi^{I}$,

$$
\mathcal{L}_{\text {Bath }}=\mathcal{R}-\frac{(\partial \phi)^{2}}{2}-V(\phi)-\frac{Y(\phi)}{2} \sum_{I=1}^{2}\left(\partial \psi^{I}\right)^{2} .
$$

When the scalar couplings are well approximated by single exponentials in the IR of the geometry,

$$
Y=e^{\alpha \phi}, \quad V=-V_{0} e^{-\beta \phi},
$$

with $\alpha, \beta$ and $V_{0}$ constants, the theory supports the following hyperscaling violating, Lifshitz-like black-branes,

$$
\begin{aligned}
d s^{2} & =-\left(\frac{r}{\ell}\right)^{2 m} f(r) d t^{2}+\left(\frac{r}{\ell}\right)^{-2 m} f(r)^{-1} d r^{2}+\left(\frac{r}{\ell}\right)^{2 n} d \vec{x}^{2}, \\
f(r) & =1-\left(\frac{r_{h}}{r}\right)^{2 m+2 n-1}, \quad \phi(r)=\kappa \ln \left(\frac{r}{\ell}\right), \\
\kappa^{2} & =4 n(1-n), \quad \alpha \kappa=2(m+n-1), \\
\beta \kappa & =2(1-m), \quad \ell^{2} V_{0}=2 m(2 m+2 n-1), \\
k^{2} & =\frac{(m-n) V_{0}}{m},
\end{aligned}
$$




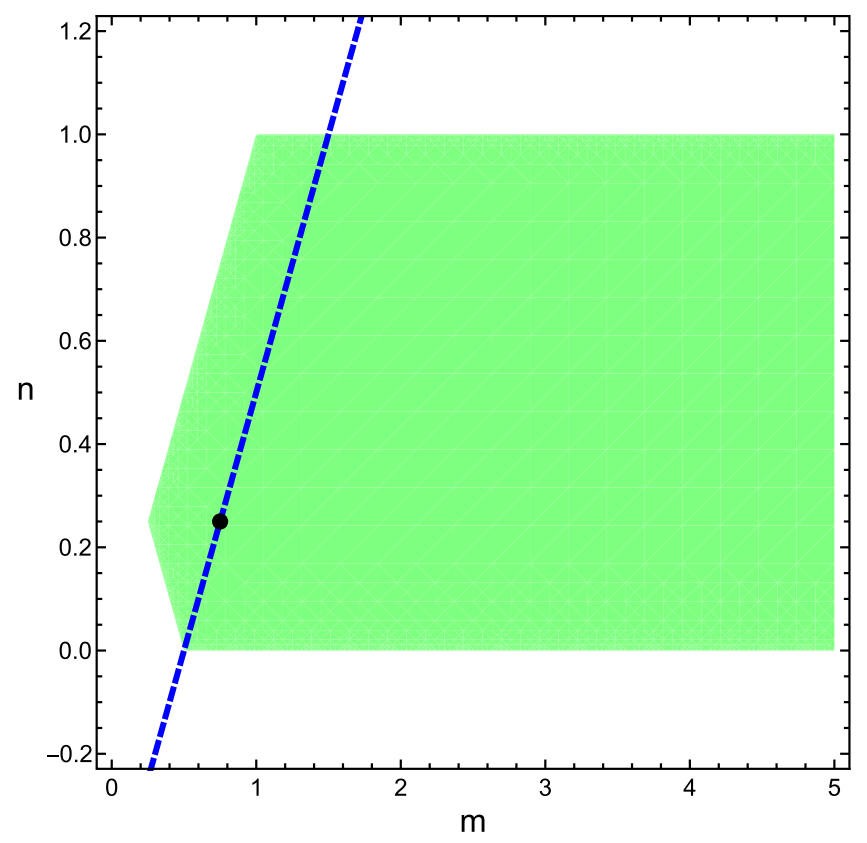

FIG. 1. The green region denotes the values of $(m, n)$ that satisfy the physical constraints which ensure a well-defined ground state geometry. The dashed blue line shows the case which gives a linear entropy $S \sim T$. The black dot corresponds to the $\eta$-geometry with $m=3 / 4, n=1 / 4$ (or $\eta=1$ ).

where we have chosen the axion configuration

$$
\psi^{1}=k x, \quad \psi^{2}=k y,
$$

with the constant $k$ denoting the strength of momentum dissipation. The temperature of these solutions scales with the horizon radius as $T \sim r_{h}^{2 m-1}$ and the entropy as $S \sim T^{\frac{2 n}{2 m-1}}$. The scaling parameters $n, m$ can be related to the standard dynamical critical exponent $z$ and hyperscaling violating exponent $\theta$ by using

$$
m=\frac{1}{2} \frac{\theta-2 z}{\theta-z}, \quad n=\frac{1}{2} \frac{\theta-2}{\theta-z} .
$$

In terms of $n, m$ the $\eta$-geometries discussed in Ref. [24], which arise from taking the limit $z \rightarrow \infty, \theta \rightarrow \infty$ with $\eta \equiv$ $-\theta / z$ held fixed, correspond to taking $n+m=1$ (with $\left.\eta=\frac{2 n}{1-2 n}\right)$. Finally, since these scaling geometries are generically singular, one needs to impose appropriate constraints on the parameter space in order to ensure a well-defined ground state solution, including Gubser's criterion [25] and the null energy condition (see e.g., the discussion in Refs. [26,27]). The parameter space corresponding to physically acceptable ranges for the exponents $(m, n)$ is shown in Fig. 1, with the dashed blue line denoting values for which the entropy is linear with temperature.

\section{A SPECIFIC NONLINEAR MODEL}

Motivated in part by the DBI analysis [13,14], we choose to focus on a particularly simple nonlinear model,

$$
\mathcal{L}(s, p, \phi)=Z(\phi) s+\frac{1}{2} Z^{2}(\phi) p^{2}
$$

characterized by a single scalar coupling $Z(\phi)$. The combination (12) is natural from the viewpoint of the DBI action

$$
\begin{aligned}
S_{\mathrm{DBI}} & =\sqrt{-g}-\sqrt{-\operatorname{det}\left(g+Z^{1 / 2} F\right)} \\
& =\sqrt{-g}\left[1-\sqrt{1-2\left(Z s+Z^{2} p^{2} / 2\right)}\right]
\end{aligned}
$$

and, despite its simplicity, will turn out to be sufficient to describe four of the scalings observed in the strange metal phase of the cuprates. Evaluating (4) for our nonlinear model (12), we find that the resistivity and Hall angle are

$$
\begin{aligned}
R_{x x} & \sim \frac{Z}{Z^{2}+Z^{4} p^{2}}=\frac{\left(C^{2}+h^{2} Z\right)^{2}}{Z\left[\left(C^{2}+h^{2} Z\right)^{2}+h^{2} \rho^{2}\right]}, \\
\cot \Theta_{H} & \sim-\frac{1}{Z p}=\frac{C^{2}+h^{2} Z}{h \rho},
\end{aligned}
$$

with the charge density

$$
\rho=\frac{A_{t}^{\prime} Z}{C}\left(C^{2}+h^{2} Z\right),
$$

and all functions evaluated at the horizon. The expressions (14) can be simplified further by taking a small $h$ limit, which is consistent with our assumption of a dilute charge sector. This yields the simple expressions

$$
R_{x x} \sim \frac{1}{Z}, \quad \cot \Theta_{H} \sim \frac{C^{2}}{h \rho},
$$

each controlled by a different scale, the first by the scalar coupling $Z$ and the second by the geometry through $C$. Finally, working with the small $h$ expansion of the resistivity (14) we compute the magnetoresistance,

$$
M R=\frac{R_{x x}(h)-R_{x x}(h=0)}{R_{x x}(h=0)} \sim-\frac{h^{2} \rho^{2}}{C^{4}} .
$$

The results (16) agree with those obtained in the DBI construction of Ref. [13], for small values of the charge density and magnetic field. While this agreement is expected, since (12) is part of the low-energy expansion of the DBI model, it also suggests that the much simpler nonlinear model (16) may suffice to capture the key physics of more complex DBI-like theories. In addition, the particular DBI model of Ref. [13] predicts a weak-field magnetoresistance that goes as $h^{2} / T^{3}$, instead of the $h^{2} / T^{4}$ behavior of the cuprates, and therefore it may be more appropriate to describe other strange metal phases. 


\section{PHYSICAL IMPLICATION}

We are now ready to comment on the implications of our results, and ask in particular whether the transport quantities we computed can describe the scaling behaviors observed in the cuprates. First of all, note that while $R_{x x}$ depends on the scalar coupling $Z$, the Hall angle and magnetoresistance (at small $h$ ) are both controlled by the metric component $C$, which also determines the thermodynamic entropy $S$ of the dual field theory, $S \sim C$. While the coupling $Z$ can be chosen freely without affecting the geometry, the function $C$ is fixed for a given background. Once the geometry is specified-and therefore the temperature dependence of the entropy-there is very little freedom in the system.

In our model a linear resistivity $R_{x x} \sim T$ can be realized by identifying a clean temperature scaling regime for the scalar coupling, of the form

$$
Z \sim \frac{1}{T} .
$$

Moreover, experimental data on the cuprates [28,29] indicates that the entropy is linear in temperature,

$$
S \sim T,
$$

which requires the spatial metric component to scale as

$$
C \sim T,
$$

and unambiguously fixes the temperature dependence of the Hall angle and magnetoresistance in our model to be

$$
\cot \Theta_{H} \sim T^{2}, \quad M R \sim-\frac{h^{2}}{T^{4}} .
$$

In our setup (18) and (20) can indeed be realized quite naturally, making use of the quantum critical geometry. In particular, (18) can be obtained by making the simple single exponential choice $Z(\phi) \sim e^{\gamma \phi}$ with $\gamma=\frac{1-2 m}{2-2 m} \beta$. Moreover, the entropy associated with (9) is given by

$$
S \sim C\left(r_{h}\right) \sim T^{\frac{2 n}{2 m-1}} .
$$

Thus, we have a linear entropy $S \sim T$ when $2 n=2 m-1$. For $z$ and $\theta$ finite this translates to the condition $z=2-\theta$ corresponding to a one-parameter family of black brane solutions, while for the case of $\eta$-geometries for which both exponents are infinite we have $m+n=1$ and thus $n=$ $1 / 4$ and $m=3 / 4$. The parameter choices that correspond to a linear entropy are represented by the dashed line in Fig. 1, with the dot denoting the special case corresponding to the $\eta=1$ geometry.

It is intriguing and unexpected that the choice (18) and the experimental observation (19) are sufficient to reproduce the observed scaling properties of the cuprates. In particular, what we have seen is that the simple nonlinear model (12) supports the following behaviors,

$S \sim T, \quad R_{x x} \sim T, \quad \cot \Theta_{H} \sim T^{2}, \quad M R \sim-\frac{h^{2}}{T^{4}}$.

It is convenient to rescale the temperature and magnetic field and work with dimensionless quantities. In particular, if we introduce two positive constants $z_{0}$ and $c_{0}$ through $Z=$ $z_{0} / T$ and $C=c_{0} T$ (the values of the constants depend on the specific theory one examines), we can construct the dimensionless expressions

$$
\mathbf{T}=\frac{c_{0}^{2} z_{0}}{\rho^{2}} T, \quad \mathbf{h}=\frac{c_{0}^{2} z_{0}^{2}}{\rho^{3}} h .
$$

We then have

$$
\begin{aligned}
R_{x x} & =\zeta_{0} \mathbf{T}\left[1+\frac{\mathbf{T}^{2} \mathbf{h}^{2}}{\left(\mathbf{T}^{3}+\mathbf{h}^{2}\right)^{2}}\right]^{-1}, \quad \zeta_{0} \equiv \frac{\rho^{2}}{c_{0}^{2} z_{0}^{2}}, \\
\cot \Theta_{H} & =\frac{\mathbf{T}^{2}}{\mathbf{h}}\left(1+\frac{\mathbf{h}^{2}}{\mathbf{T}^{3}}\right), \quad M R=-\frac{\mathbf{T}^{2} \mathbf{h}^{2}}{\left(\mathbf{T}^{3}+\mathbf{h}^{2}\right)^{2}+\mathbf{T}^{2} \mathbf{h}^{2}} .
\end{aligned}
$$

We immediately observe that $R_{x x} \sim \mathbf{T}$ approaches zero as $\mathbf{T} \rightarrow 0$, and in particular $R_{x x}=\zeta_{0} \mathbf{T}$ in the absence of a magnetic field. Thus, this system indeed describes a metal phase.

The temperature dependence of the quantities (25) is plotted in Fig. 2, from which it is clear that when the value of $\mathbf{T}$ is sufficiently bigger than $\mathbf{h}$ (more precisely, when $\mathbf{T}^{3} \gg \mathbf{h}^{2}$ ), one realizes the strange metal scalings

$$
S \sim \mathbf{T}, \quad R_{x x} \sim \zeta_{0} \mathbf{T}, \quad \cot \Theta_{H} \sim \frac{\mathbf{T}^{2}}{\mathbf{h}}, \quad M R \sim-\frac{\mathbf{h}^{2}}{\mathbf{T}^{4}} .
$$

Our discussion is based on the rescaled temperature $\mathbf{T}$ which is defined with respect to the scale $\rho^{2} /\left(c_{0}^{2} z_{0}^{2}\right)$, as seen from (24). Therefore, note that the regime we are considering is not necessarily a high- $T$ limit. It could indeed describe low temperature physics provided that this scale is sufficiently higher than the temperature the experiment is probing.

In closing, we would like to mention a few additional features that are visible in our analysis. First, note that in the absence of a magnetic field the linear temperature dependence of the resistivity is exact. Moreover, as long as we are away from the transition regime in which $\mathbf{T}$ and $\mathbf{h}$ are comparable, the in-plane resistivity (25) is not very sensitive to the magnetic field. As a result, in the temperature range in 

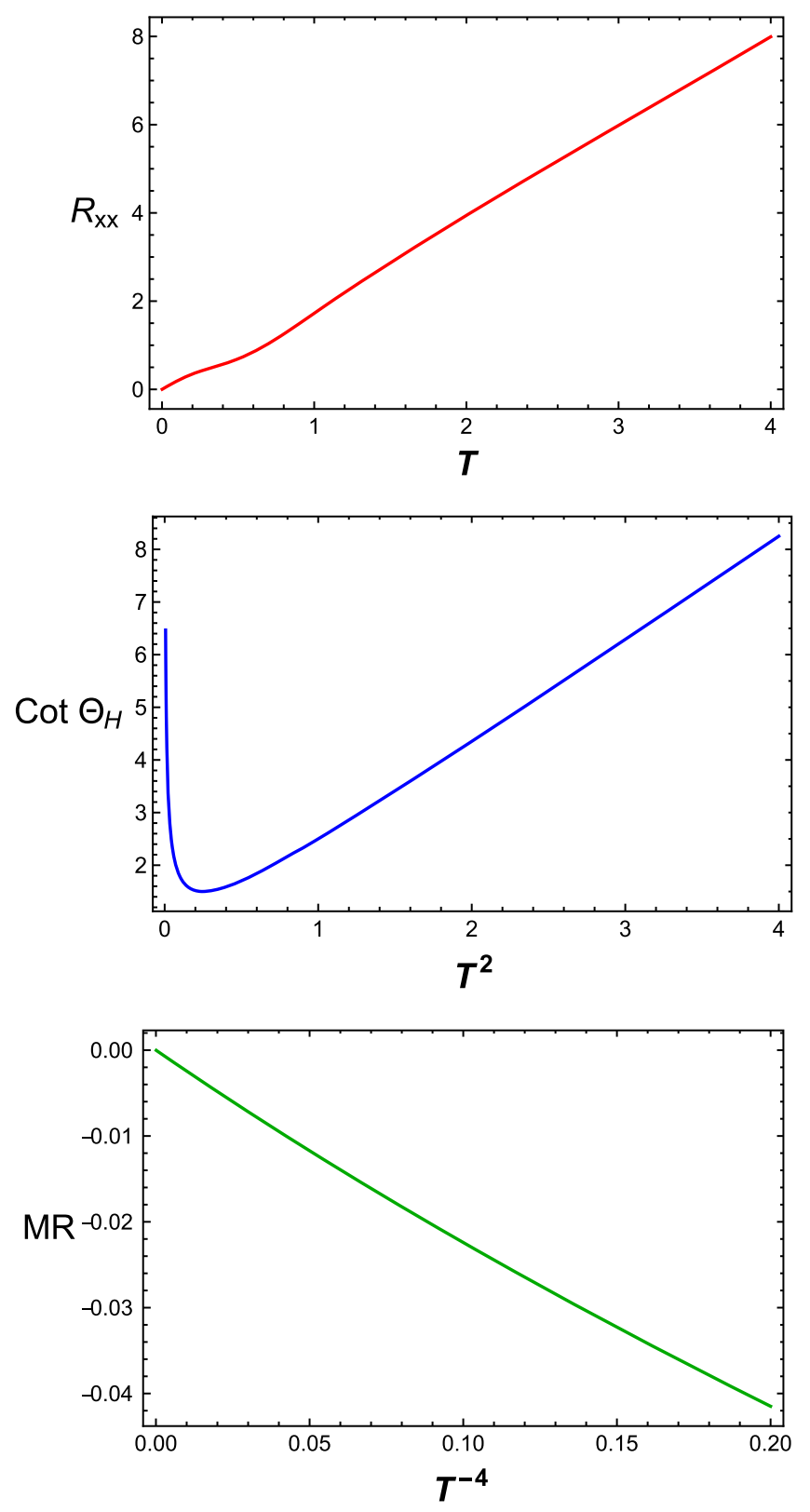

FIG. 2. Dependence of the resistivity, Hall angle and magnetoresistance in the expressions (25) on the dimensionless temperature $\mathbf{T}$. We fix $\mathbf{h}=0.5$ and find good scaling behaviors when $\mathbf{T} \gtrsim 3 \mathbf{h}=1.5$. We have chosen $\zeta_{0}=2$.

which one can realize (26), the magnetoresistance is small (and negative), suggesting that the effect of a magnetic field does not alter the underlying normal state. This is consistent with the observation in the experiment of Ref. [30]. Finally, we note that the increase in the Hall angle at very small temperatures (visible in Fig. 2) is similar to the behavior observed in Refs. [31,32].

\section{FINAL REMARKS}

Our analysis has provided the first holographic realization of the temperature scalings (26) through a simple and rather minimal nonlinear model. In particular, our results relied crucially on the presence of nonlinear interactions among the charge carriers, which appear to be a necessary ingredient for a bulk description of strongly correlated electron matter. Working in the dilute charge limit allowed us to construct a neutral quantum critical bath, key to identifying clean scaling regimes in the transport coefficients.

In the model (12) the scaling of the Hall angle and of the magnetoresistance, $T^{2}$ and $T^{-4}$ respectively, were entirely fixed by the observation that the entropy in the cuprates should be linear in temperature [28,29], $S \sim T$. Note that the linear resistivity $R_{x x} \sim T$ would then follow immediately by requiring $R_{x x}$ to scale like the entropy, making the choice (18) natural. Although $[33,34]$ argues that the resistivity should indeed be proportional to the entropy, their arguments are not applicable in our model-the inclusion of axions leads to a temperature dependent shear viscosity to entropy ratio [35]. Nonetheless, our study lends evidence to the idea that the cuprates' strange metal behavior depends crucially on the linear entropy as well as on the existence of a strongly coupled quantum critical IR sector.

Note that the magnetoresistance associated with (12) is negative. Possible mechanisms for a negative magnetoresistance have been proposed e.g., in Refs. [36-38]. We should stress, however, that both the sign and scaling properties of quantities such as the magnetoresistance depend entirely on the specific model one works with (as an example we refer the reader to the DBI analysis in the Supplemental Material [18]). Indeed, the minimal model (12) is only one among a large class of nonlinear theories one could construct, which generically lead to a highly nontrivial transport structure, as shown in (6) (and in (S26) away from the probe limit in the Supplemental Material [18]). Understanding this rich structure in more detail is especially important given the additional scaling regimes that have been recently observed in different high- $\mathrm{T}_{c}$ superconductors, including the cuprates [39] and also iron pnictides [40].

With this in mind, we hope that our results can provide guidance towards the construction of more realistic theories and help build intuition for the mechanisms underlying the unconventional behavior of the cuprates. Since the bulk nonlinearities are related to operator product expansion coefficients of the dual CFT, it would be interesting if the bulk analysis could be used to constrain the structure of the dual field theory and provide physical intuition. The question remains to what extent such nonlinear holographic models can capture the universality of the strange metal phase. It would be desirable to have a more basic understanding of the role of nonlinear effects on transport. For example, it would be valuable to identify physical regimes in which additional nonlinear terms (coming e.g., from further expanding the DBI action) are subdominant and can be neglected. That would serve to better motivate the minimal model we have studied in this paper. 


\section{ACKNOWLEDGMENTS}

We would like to thank Sean Hartnoll, Chris Pope, Mike Blake and Richard Davison for valuable conversations. The work of S. C., A. H. and S. W. is supported in part by the National Science Foundation Grant No. PHY-1620169.

[1] B. Keimer, S. A. Kivelson, M. R. Norman, S. Uchida, and J. Zaanen, High temperature superconductivity in the cuprates, Nature (London) 518, 179 (2015).

[2] J. M. Harris, Y. F. Yan, P. Matl, N. P. Ong, P. W. Anderson, T. Kimura, and K. Kitazawa, Violation of Kohler's Rule in the Normal-State Magnetoresistance of $\mathrm{YBa}_{2} \mathrm{Cu}_{3} \mathrm{O}_{7-\delta}$ and $\mathrm{La}_{2} \mathrm{Sr}_{x} \mathrm{CuO}_{4}$, Phys. Rev. Lett. 75, 1391 (1995).

[3] N. E. Hussey, Phenomenology of the normal state in-plane transport properties of high- $T_{c}$ cuprates, J. Phys. Condens. Matter 20, 123201 (2008).

[4] S. A. Hartnoll and A. Karch, Scaling theory of the cuprate strange metals, Phys. Rev. B 91, 155126 (2015).

[5] J. Zaanen, Y. W. Sun, Y. Liu, and S. Schalm, Holographic Duality in Condensed Matter Physics (Cambridge University Press, Cambridge, England, 2015).

[6] S. A. Hartnoll, A. Lucas, and S. Sachdev, Holographic quantum matter, arXiv:1612.07324.

[7] M. Blake and A. Donos, Quantum Critical Transport and the Hall Angle in Holographic Models, Phys. Rev. Lett. 114, 021601 (2015).

[8] A. Amoretti and D. Musso, Magneto-transport from momentum dissipating holography, J. High Energy Phys. 09 (2015) 094.

[9] Z. Zhou, J. P. Wu, and Y. Ling, DC and Hall conductivity in holographic massive Einstein-Maxwell-Dilaton gravity, J. High Energy Phys. 08 (2015) 067.

[10] A. Amoretti, M. Baggioli, N. Magnoli, and D. Musso, Chasing the cuprates with dilatonic dyons, J. High Energy Phys. 06 (2016) 113.

[11] B. S. Kim, E. Kiritsis, and C. Panagopoulos, Holographic quantum criticality and strange metal transport, New J. Phys. 14, 043045 (2012).

[12] A. Karch, Conductivities for hyperscaling violating geometries, J. High Energy Phys. 06 (2014) 140.

[13] E. Blauvelt, S. Cremonini, A. Hoover, L. Li, and S. Waskie, Holographic model for the anomalous scalings of the cuprates, Phys. Rev. D 97, 061901 (2018).

[14] S. A. Hartnoll, J. Polchinski, E. Silverstein, and D. Tong, Towards strange metallic holography, J. High Energy Phys. 04 (2010) 120.

[15] E. Kiritsis and L. Li, Quantum criticality and DBI magnetoresistance, J. Phys. A 50, 115402 (2017).

[16] X. Guo, P. Wang, and H. Yang, Membrane paradigm and holographic DC conductivity for nonlinear electrodynamics, Phys. Rev. D 98, 026021 (2018).

[17] N. Iqbal and H. Liu, Universality of the hydrodynamic limit in AdS/CFT and the membrane paradigm, Phys. Rev. D 79, 025023 (2009).

[18] See Supplemental Material at http://link.aps.org/ supplemental/10.1103/PhysRevD.99.061901 for a discussion of holographic conductivities with a generic gauge sector and for magnetotransport in the DBI model. It supports statements made in the paper and includes Refs. [7,13,15,19,20].

[19] S. Cremonini, A. Hoover, and L. Li, Backreacted DBI magnetotransport with momentum dissipation, J. High Energy Phys. 10 (2017) 133.

[20] P. Wang, H. Wu, and H. Yang, Holographic DC conductivity for backreacted nonlinear electrodynamics with momentum dissipation, Eur. Phys. J. C 79, 6 (2019).

[21] A. L. Fitzpatrick, S. Kachru, J. Kaplan, and S. Raghu, NonFermi liquid fixed point in a Wilsonian theory of quantum critical metals, Phys. Rev. B 88, 125116 (2013).

[22] A. L. Fitzpatrick, S. Kachru, J. Kaplan, and S. Raghu, NonFermi-liquid behavior of large- $N_{B}$ quantum critical metals, Phys. Rev. B 89, 165114 (2014).

[23] S. A. Hartnoll, R. Mahajan, M. Punk, and S. Sachdev, Transport near the Ising-nematic quantum critical point of metals in two dimensions, Phys. Rev. B 89, 155130 (2014).

[24] S. A. Hartnoll and E. Shaghoulian, Spectral weight in holographic scaling geometries, J. High Energy Phys. 07 (2012) 078.

[25] S. S. Gubser, Curvature singularities: The good, the bad, and the naked, Adv. Theor. Math. Phys. 4, 679 (2000).

[26] C. Charmousis, B. Gouteraux, B. S. Kim, E. Kiritsis, and R. Meyer, Effective holographic theories for low-temperature condensed matter systems, J. High Energy Phys. 11 (2010) 151.

[27] S. Cremonini and L. Li, Criteria for superfluid instabilities of geometries with hyperscaling violation, J. High Energy Phys. 11 (2016) 137.

[28] J. W. Loram, K. A. Mirza, J. R. Cooper, and W. Y. Liang, Electronic Specific Heat of $\mathrm{YBa}_{2} \mathrm{Cu}_{3} \mathrm{O}_{6+x}$ from 1.8 to 300 K, Phys. Rev. Lett. 71, 1740 (1993).

[29] J. W. Loram, J. Luo, J. R. Cooper, W. Y. Liang, and J. L. Tallon, Evidence on the pseudogap and condensate from the electronic specific heat, J. Phys. Chem. Sol. 62, 59 (2001).

[30] R. Daou et al., Linear-T resistivity and change in Fermi surface at the pseudogap critical point of a high- $T_{c}$ superconductor, Nat. Phys. 5, 31 (2009).

[31] T. F. Rosenbaum, A. Husmann, S. A. Carter, and J. M. Honig, Temperature dependence of the Hall angle in a correlated three-dimensional metal, Phys. Rev. B 57, R13997(R) (1998).

[32] Z. Konstantinovic, Z.Z. Li, and H. Raffy, Temperature dependence of the Hall angle in single-layer and bilayer $\mathrm{Bi}_{2} \mathrm{Sr}_{2} \mathrm{Ca}_{n-1} \mathrm{Cu}_{n} \mathrm{O}_{y}$ thin films at various oxygen contents, Phys. Rev. B 62, R11989 (2000). 
[33] R. A. Davison, K. Schalm, and J. Zaanen, Holographic duality and the resistivity of strange metals, Phys. Rev. B 89, 245116 (2014).

[34] J. Zaanen, Planckian dissipation, minimal viscosity and the transport in cuprate strange metals, arXiv:1807.10951.

[35] S. A. Hartnoll, D. M. Ramirez, and J. E. Santos, Entropy production, viscosity bounds and bumpy black holes, J. High Energy Phys. 03 (2016) 170.

[36] P. Fournier, J. Higgins, H. Balci, E. Maiser, C. J. Lobb, and R. L. Greene, Anomalous saturation of the phase coherence length in underdoped $\mathrm{Pr}_{2-x} \mathrm{Ce}_{x} \mathrm{CuO}_{4}$ thin films, Phys. Rev. B 62, R11993(R) (2000).

[37] T. Sekitani, M. Naito, and N. Miura, Kondo effect in underdoped $n$-type superconductors, Phys. Rev. B 67, 174503 (2003).
[38] I. M. Hayes, Z. Hao, N. Maksimovic, S. K. Lewin, M. K. Chan, R. D. McDonald, B. J. Ramshaw, J.E. Moore, and J.G. Analytis, Magnetoresistance Scaling Reveals Symmetries of the Strongly Correlated Dynamics in $\mathrm{BaFe}_{2}\left(\mathrm{As}_{1-x} \mathrm{P}_{x}\right)_{2}$, Phys. Rev. Lett. 121, 197002 (2018).

[39] P.-G. Gallo et al., Scale-invariant magnetoresistance in a cuprate superconductor, Science 361, 479 (2018).

[40] I. M. Hayes, R. D. McDonald, N. P. Breznay, T. Helm, P. J. W. Moll, M. Wartenbe, A. Shekhter, and J. G. Analytis, Scaling between magnetic field and temperature in the hightemperature superconductor $\mathrm{BaFe}_{2}\left(\mathrm{As}_{1-x} \mathrm{P}_{x}\right)_{2}$, Nat. Phys. 12, 916 (2016). 